[Article]

www.whxb.pku.edu.cn

\title{
超声辅助法制备双孔空心球状碳化铇及其电催化性能
}

\author{
陈赵扬赵峰鸣 马淳安* 乔 芸 \\ (浙江工业大学化工材料学院, 绿色化学合成技术国家重点实验室培育基地, 科技部能源材料及 \\ 应用国际科技合作基地,杭州 310032)
}

\begin{abstract}
摘要：采用超声空化辅助法制得空心球状结构偏铇酸铵(AMT), 通过固定床气-固反应, 以 $\mathrm{CO} / \mathrm{H}_{2}$ 为还原碳化气 氛在700-900 ${ }^{\circ} \mathrm{C}$ 下制备得到了介孔结构空心球状碳化铇. 并再次超声处理后分解球型结构得到条状介孔碳化铇. 用扫描电镜(SEM)、X射线衍射(XRD)、热重-差热分析(TG-DTA)、比表面测试(BET)和孔径分布测试(BJH)对样品 进行了表征. 结果表明, 样品组成为单相碳化铇, 且在空气中低于 $410{ }^{\circ} \mathrm{C}$ 时具有高稳定性并且具有 4 和 $22 \mathrm{~nm}$ 的双 孔隙分布结构. 将碳化铇粉末制成粉末微电极 (PME), 采用循环伏安 (CV)技术研究了碳化铇在质子惰性介质中 对硝基苯的电催化还原性能. 结果表明, 相比铂微盘电极, 双孔结构碳化铇对硝基苯还原有着更好的电催化活 性, 在峰电位上比铂微盘正移了 $30 \mathrm{mV}, I_{\mathrm{p}}-v^{12}$ 关系曲线显示硝基苯在粉末微电极中的还原行为受扩散控制.
\end{abstract}

关键词：碳化铇; 超声辅助; 介孔; 电催化

中图分类号：0646

\section{Ultrasonic-Assisted Preparation of Bimodal Mesoporous Hollow Global Tungsten Carbide and Its Electrocatalytic Performance}

\author{
CHEN Zhao-Yang ZHAO Feng-Ming MA Chun-An* QIAO Yun \\ (State Key Laboratory Breeding Base for Green Chemistry Synthesis Technology, International Science \& Technology \\ Cooperation Base of Energy Materials and Application, College of Chemical Engineering and \\ Materials Science, Zhejiang University of Technology, Hangzhou 310032, P. R. China)
}

\begin{abstract}
Hollow spherical ammonium metatungstate (AMT), as a precursor, was prepared by an ultrasonic method. Tungsten carbide (WC) was prepared by a gas-solid reaction in an atmosphere of $\mathrm{CO} / \mathrm{H}_{2}$ at $700-900{ }^{\circ} \mathrm{C}$. Microspheres were fractured by ultrasonic dispersion for $1 \mathrm{~h}$. X-ray diffraction (XRD), scanning electron microscopy (SEM), thermogravimetry-differential thermal analysis (TG-DTA), Brunauer-Emmett-Teller (BET) surface area, and BarrettJoyner-Halenda $(\mathrm{BJH})$ pore-size distribution were used to characterize the morphology, mesoporous structure, and thermal stability of the sample. The results indicate that the sample is pure WC. The WC sample is stable in air at $410{ }^{\circ} \mathrm{C}$ and the mesopores of WC were centered at $4 \mathrm{~nm}$ and $22 \mathrm{~nm}$. A WC powder microelectrode (WC-PME) was prepared using the prepared WC powders. The activity of WC for the electroreduction of nitrobenzene was studied by cyclic voltammetry $(\mathrm{CV})$. The results indicate that the bimodal porosity of WC-PME led to higher catalytic activity than that of a Pt micro disc electrode (Pt-MDE). The reduction potential was $30 \mathrm{mV}$ more positive than that of the Pt-MDE. The relation $I_{\mathrm{p}}-v^{1 / 2}$ showed that the electrode reaction was controlled by liquid diffusion.
\end{abstract}

Key Words: Tungsten carbide; Ultrasonic-assistance; Mesoporous; Electrocatalysis

Received: January 15, 2010; Revised: May 9, 2010; Published on Web: July 9, 2010.

"Corresponding author. Email: science@zjut.edu.cn; Tel: +86-571-88320360.

The project was supported by the Science and Technology Major Project in International Cooperation of Zhejiang Province, China (2008Cl4040), Key Project of Natural Science Foundation of Zhejiang Province, China (Z4100790) and National Natural Science Foundation of China (20476097). 浙江省重大科技专项国际合作项目(2008Cl4040), 浙江省自然科学基金重点项目(Z4100790)和国家自然科学基金(20476097)资助

(C) Editorial office of Acta Physico-Chimica Sinica 
近年来, 碳化铇(WC)由于拥有众多的应用领域 而备受关注, 尤其在催化领域, 其类铂催化性能更是 受到广泛探究 ${ }^{[1-3]}$. 从已发表的论文上看, $\mathrm{WC}$ 在化学 催化领域可用作加氢、异构化和烃类转化等反应 的催化剂 ${ }^{[4]}$. 在电化学领域, WC 更是在氢离子化和 析氢反应中表现出了良好的性能 ${ }^{[-8]}$. 除此以外, WC 由于延续了硬质合金应用的优良属性, 其良好的稳 定性得到了广泛的肯定, 从而也为 WC 作为高性能 催化剂奠定了基础. 在 WC 催化剂设计方面, 纳米 催化剂和多孔催化剂已经成为一个主要的趋势, 对 于 WC 来讲, 通常情况下, 由于其高稳定性和高硬 度特性使得在成相后的再改造困难较大, 如对其球 磨所需的能耗就相对较高. 所以需要对其前驱物质 进行结构设计. 此外, 在还原碳化步骤中, 还原性气 体碳氢化合物的种类和组成比例会影响产品的结构 和微观化学组成 ${ }^{[9-10]}$, 从而影响 WC 的选择性及催 化活性 ${ }^{[11-13]}$. 上述研究表明, 特殊结构和不同物相组 成的 WC 催化剂的制备具有十分重要的理论研究 意义和实际应用价值. 因而, 如何对其前驱体的组成 和结构在碳化过程中进行控制的研究显得尤为重 要.

偏铇酸铵 (AMT) 作为使用最广泛的 WC 前驱体 有着很多优点. 但常规的 AMT 颗粒较大, 有的甚至 达到毫米级, 而碳化后的 WC 基本保持了原有颗粒 大小和形状. 如何使用实验室常规仪器对 AMT 进 行再造粒的研究可使得高性能碳化铇生产成本大幅 度降低.

超声空化设备购买方便和廉价, 且具有低能耗、 无污染等特点, 在结晶、分散、萃取和电镀等化工和 环保领域得到了广泛应用 ${ }^{[14-15]}$, 但在纳米和介孔材 料制备上却少有报道. 如果能将其利用到 WC 前驱 体制备上来, 将有较大的实用意义和可行性. 其超声 空化作用产生的微泡有望瞬间对前驱体进行再造 粒, 从而有效改善 WC 结构和性能.

本研究使用超声辅助方法, 利用 AMT 的高水 溶性和不溶于乙醇的特点, 使用无水乙醇作为沉淀 剂在超声波中对 AMT 水溶液进行瞬间造粒和分散. 并使用富氢气氛对前驱体进行还原碳化. 因芳香族 硝基化合物的电还原反应是有机电化学领域中一类 重要的绿色化学反应, 因此, 样品制成电极后, 初步 评价了 $\mathrm{WC}$ 在质子惰性体系下对硝基苯( $\mathrm{NB}$ ) 的电 催化还原性能.

\section{1 实验部分}

\section{1 原料与仪器}

偏铇酸铵 $\left(\left(\mathrm{NH}_{4}\right)_{6}\left(\mathrm{H}_{2} \mathrm{~W}_{12} \mathrm{O}_{40}\right) \cdot 4 \mathrm{H}_{2} \mathrm{O}\right), \mathrm{WO}_{3} \geqslant 89 \%$, 工业级, 姜堰市贝斯特钿制品有限公司; 乙醇 $(\mathrm{EtOH})$, 分析纯, 安徽安特生物化学有限公司; 硝基 苯 $\left(\mathrm{C}_{6} \mathrm{H}_{5} \mathrm{NO}_{2}, \mathrm{NB}\right)$, 分析纯, 上海化学试剂公司; 四丁 基高氯酸铵 $\left(\mathrm{C}_{10} \mathrm{H}_{36} \mathrm{NClO}_{4}, \mathrm{TBAP}\right)$, 含量 $98 \%$, 武汉金金 华远科技发展有限公司; $N, N$-二甲基甲酰胺(DMF), 分析纯, 如臬市金陵试剂厂; $\mathrm{H}_{2}, \varphi \geqslant 99.99 \%, \mathrm{CO}$, $\varphi \geqslant 99.99 \%, \mathrm{~N}_{2}, \varphi \geqslant 99.5 \%$, 杭州今工气体. 实验用水 为自制一次蒸馏水.

超声清洗机(EHSY, $50 \mathrm{kHz}$ ), 实验加热设备采 用上海电炉厂生产的管式电阻炉(上海实验电炉厂 SK2-2-10 型), 加温设备采用温度控制器(厦门宇电), 气体流量控制采用质量流量计(北京七星华创).

\section{2 样品的制备}

图 1 为前驱体制备装置示意图. 如图 1 所示, 取 100-200 mL 的无水乙醇, 超声振荡. 在 $10 \mathrm{~mL}$ 去离 子水中加人 20-25 g AMT, 超声搅拌至完全溶解后, 移人微量进样管中缓慢地将 AMT 水溶液滴加至超 声振荡的无水乙醇中. 待全部滴加完毕后得到白色 固体沉淀. 经过滤后, 在 $50{ }^{\circ} \mathrm{C}$ 恒温下真空干燥, 得到 白色粉末, 装瓶后放人干燥器内待用.

将干燥后的 AMT 颗粒装人石英舟内, 放人管 式反应炉, 管式炉内通人 $\mathrm{CO}$ 和 $\mathrm{H}_{2}$ 混合气体, 采用 程序升温方式, 首先将温度升高到 $400{ }^{\circ} \mathrm{C}$, 保温 1-2 $\mathrm{h}$, 再升温到 $800{ }^{\circ} \mathrm{C}$ 保温 $6 \mathrm{~h}$, 之后在 $1 \mathrm{~h}$ 内将样品温 度在氮气保护下降至室温后取出. 将部分样品进行 超声再分散后干燥保存. 采用 XRD、SEM 和 TGDTA 等方法对样品进行表征.

\section{3 表征方法}

XRD(Thremo ARL SCINTAG X"TRA), Cu $K_{\alpha}$ 靶, 管电流 $40 \mathrm{~mA}$, 管电压 $45 \mathrm{kV}$, 步长 $0.04^{\circ}$, 扫描

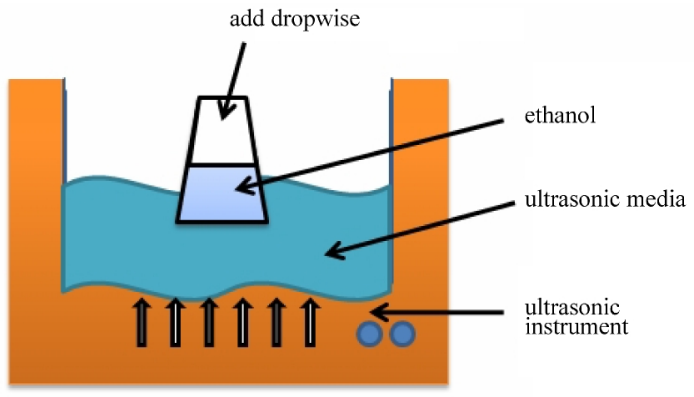

图 1 前驱体制备装置示意图

Fig.1 Schematic diagram of the experimental setup 
速率 $2.4\left(^{\circ}\right) \cdot \mathrm{min}^{-1}$, 扫描范围 $15^{\circ}-80^{\circ}$. 样品形貌与结 构分析采用 SEM(日本 Hitachi S-4700II)表征. 使用 TG-DTA(Seiko Instruments) 测定样品的热稳定性, 将约 $10 \mathrm{mg}$ 样品置于 $\alpha-\mathrm{Al}_{2} \mathrm{O}_{3}$ 坩埚中, 在流速为 100 $\mathrm{mL} \cdot \mathrm{min}^{-1}$ 空气气氛保护下, 以 $10^{\circ} \mathrm{C} \cdot \mathrm{min}^{-1}$ 的升温速 率, 在 40-1000 ${ }^{\circ} \mathrm{C}$ 温度范围内测定样品的热力学 性质.

\section{4 电化学测试}

电化学测试所用的仪器为 CHI660C 型电化学 工作站, 测试过程在三电极体系中进行. 工作电极为 填充了 WC 样品的粉末微电极(WC-PME), 微电极 微孔孔径为 $60 \mu \mathrm{m}^{[16]}$, 参比电极为饱和甘永电极 (SCE), 对电极为大面积 $(20 \mathrm{~mm} \times 10 \mathrm{~mm}) \mathrm{Pt}$ 片. 在本 文中提到的电位均相对于 SCE.

阴极液为含硝基苯 $(\mathrm{NB})\left(0.03 \mathrm{~mol} \cdot \mathrm{L}^{-1}\right)$ 的四丁基 高氯酸铵 $(\mathrm{TBAP})\left(0.1 \mathrm{~mol} \cdot \mathrm{L}^{-1}\right)$ 的 $N, N$-二甲基甲酰胺 (DMF) 溶液. 阴阳极室间用多孔烧结玻璃分隔. 所用 试剂均为分析纯. 实验测试在 $(288.0 \pm 0.1) \mathrm{K}$ 下进 行. 在测试之前电解液先鼓 $\mathrm{N}_{2} 30 \mathrm{~min}$, 以除去电解 液中的溶解氧.

\section{2 结果与讨论}

\section{1 样品物相分析}

图 2 为 $\mathrm{WC}$ 样品的 XRD 分析结果. 从图中可
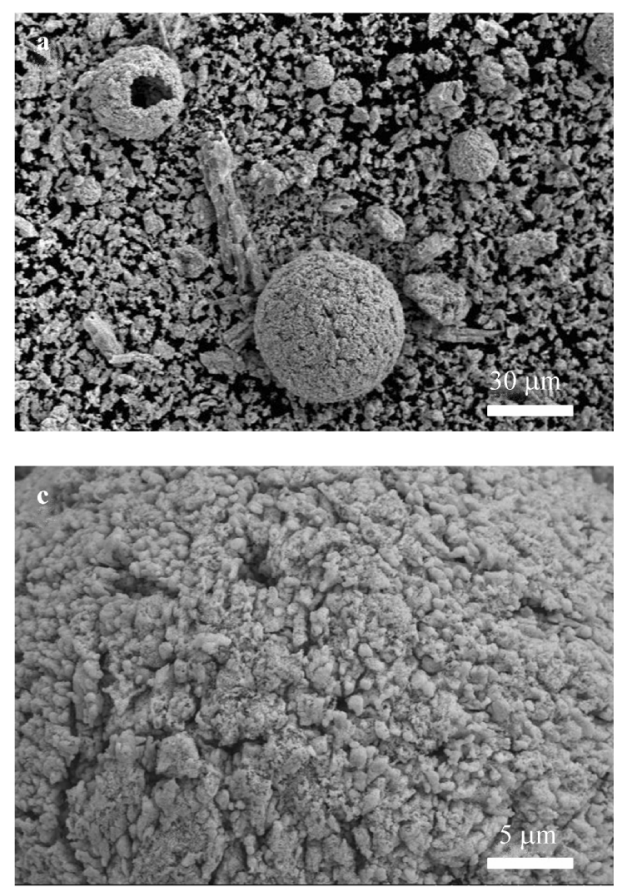

图 3 不同放大倍数的介孔空心球状 WC 的 SEM 图
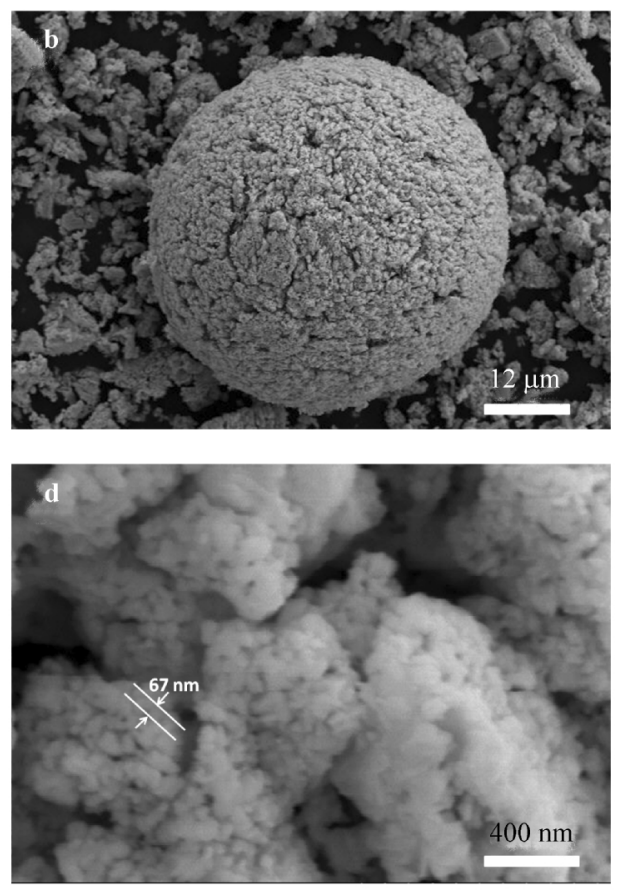

以看出, $2 \theta$ 在 $31.26^{\circ} 、 35.64^{\circ} 、 48.25^{\circ}$ 处有 3 个最强的 衍射峰, 分别对应于 WC 的(001)、(100)和(101)三个 晶面; 此外, 有几处相对较弱的衍射峰, 其 $2 \theta$ 值依次 为 $64.61^{\circ} 、 65.28^{\circ} 、 73.24^{\circ} 、 75.73^{\circ}$ 和 $76.88^{\circ}$, 分别对应 $\mathrm{WC}$ 的(110)、(002)、(111)、(200)和(102)晶面, $2 \theta$ 值 与 JCPDS25-1047 标准数据的 $2 \theta$ 值相比基本一致, 但稍微有点差异, 说明样品的物相组成以 WC 为主, 且 WC 呈正六方结构, 上述差异可能由于碳化过程 中富氢比例气体的存在使得积碳被有效去除, 而样 品降温后吸附了微量氧所致.

\section{2 样品结构与形貌}

WC 样品的 SEM 表征结果如图 3 所示, 图 3a

Fig.3 SEM images of mesoporous WC ball with different magnifications 

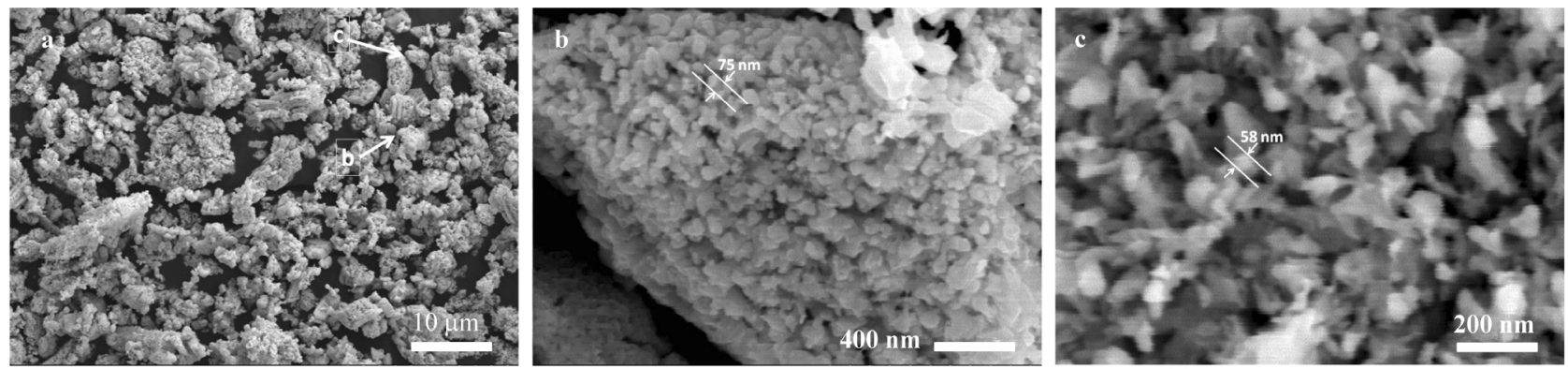

图 4 经 $1 \mathrm{~h}$ 超声分散后的 WC 样品不同放大倍数的 SEM 图

Fig.4 SEM images of WC sample after $1 \mathrm{~h}$ supersonic treatment with different magnifications

为样品的总体形貌, 可以看到样品分布了大量的球 状 $\mathrm{WC}$, 且从左上角的破碎球体中可以看到空心结 构 WC 的存在, 其形成原因可能是微量高浓度偏铇 酸铵溶液滴加到无水乙醇中以后, 因为偏铇酸铵不 溶于乙醇, 所以在进人乙醇相以后, 由于偏铇酸铵达 到过饱和状态在瞬间产生沉淀. 而在此时, 无水乙醇 正由于在超声介质中的高频机械振动产生数以万计 的微小气泡, 这些气泡在超声波纵向传播的负压区 形成、生长. 从而得到了空心球状偏铇酸铵沉淀. 从 图中可以看到, $\mathrm{WC}$ 球体大小分布不均, 这可能与滴 加瞬间产生沉淀时候的空化气泡大小有关. 从图 3 $(b, c)$ 可以看出, 球体表面并非光滑的 WC 结构, 而 是充满了孔隙结构.

在进一步放大的 SEM 图(图 3d)中可以看出, 球 体结构的组成由纳米 WC 颗粒组成, 而且 WC 微粒 有两种组成方式, 其中从大颗粒上看, 介孔空心球状 $\mathrm{WC}$ 由 $500 \mathrm{~nm}$ 左右的大颗粒组成, 而这些大颗粒的 $\mathrm{WC}$ 又是由纳米 WC 组成. 纳米 WC 颗粒大小介于 50-70 nm 之间. 从连接方式上看, 大颗粒间组合呈 无规则状态, 也可能结合力上有少许减低. 而纳米颗 粒分布相对规则, 孔隙分布均匀.

为了得到更细、分布更加均匀的 WC 样品, 我 们对介孔空心球状 WC 样品进行了 $1 \mathrm{~h}$ 的超声分散 处理. 得到样品的 SEM 图如图 4 所示, 球状 WC 消 失, 取而代之的是条状的 $\mathrm{WC}$ 碎片, 碎片大小基本 处于 3-5 $\mu \mathrm{m}$ 之间. 图 4(b,c) 中碎片颗粒由纳米 WC 组成, 纳米颗粒间组成了孔隙结构, 孔隙基本在 40 $\mathrm{nm}$ 以下, 属于基本介孔范畴. 从颗粒表面和断面上 可以看出, 孔隙之间相互连通, 前后贯通. 这样的结 构更有利于在催化反应中的反应物传质.

\section{3 热稳定性}

很多催化反应都需要适当加温, 合适的 WC 催 化剂需要在一定温度范围内保持稳定的性能. 图 5
为 $\mathrm{WC}$ 样品在空气气氛下的热重-差热分析 (TGDTA)结果. 从图中的 $\mathrm{TG}$ 曲线可以看出, 在 $410{ }^{\circ} \mathrm{C}$ 时样品开始有明显的增重现象, 到 $530{ }^{\circ} \mathrm{C}$ 左右样品 重量基本保持稳定, 相应在 DTA 曲线中, 410-530 ${ }^{\circ} \mathrm{C}$ 之间存在放热现象. 从 DTA 曲线中还可以发现 2 个放热峰, 这可能是由于样品存在两种粒径或两种 孔径. 期间大的放热峰放热温度区间较小, 说明小颗 粒 $\mathrm{WC}$ 氧化较早, 而之后少部分硬团聚 $\mathrm{WC}$ 缓慢氧 化. 总的来说, 在空气氛围中, 当温度低于 $410{ }^{\circ} \mathrm{C}$ 时 样品是稳定的. $410{ }^{\circ} \mathrm{C}$ 以后, 样品将与空气中的氧发 生氧化反应, 同时放出热量; 当氧化反应完全后, 即 碳全被氧原子取代时, 样品的重量保持稳定.

\section{4 样品的孔隙分布}

从氮气吸脱附的测试结果中可以计算出样品的 孔径分布. 图 6 给出了 $\mathrm{WC}$ 样品的低温 $\mathrm{N}_{2}$ 吸附-脱 附曲线及计算得到的孔径分布结果. 从图 6(a) 可以 看出, 介孔 $\mathrm{WC}$ 的吸附-脱附曲线在相对压力 $0.8-$ 1.0 范围内上升较大, 说明有介孔存在. 图6(b)的孔 径分布曲线呈现 2 个主峰, 较小的是 $4 \mathrm{~nm}$ 处的窄而 尖的峰,而 $22 \mathrm{~nm}$ 处的峰位表现了第二种孔的存在,

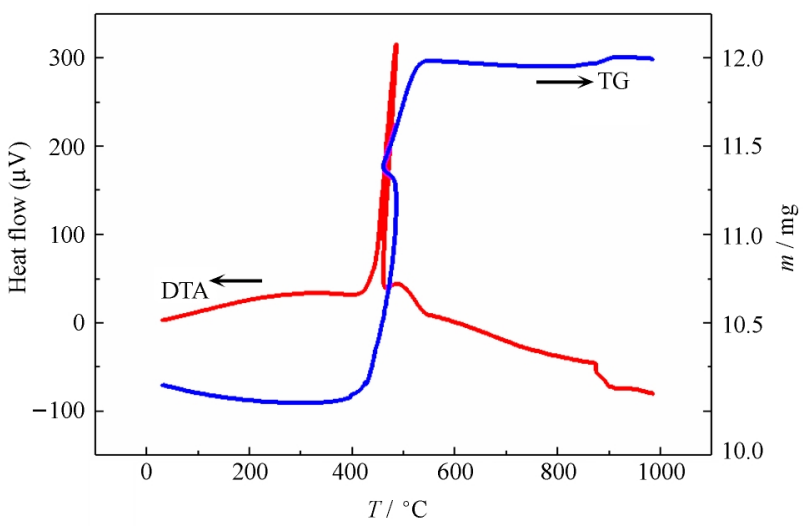

图 5 WC 样品在空气中的 TG-DTA 曲线

Fig.5 TG-DTA curves of WC sample heating rate: $10{ }^{\circ} \mathrm{C} \cdot \mathrm{min}^{-1}$, atmosphere: air, $100 \mathrm{~mL} \cdot \mathrm{min}^{-1}$ 

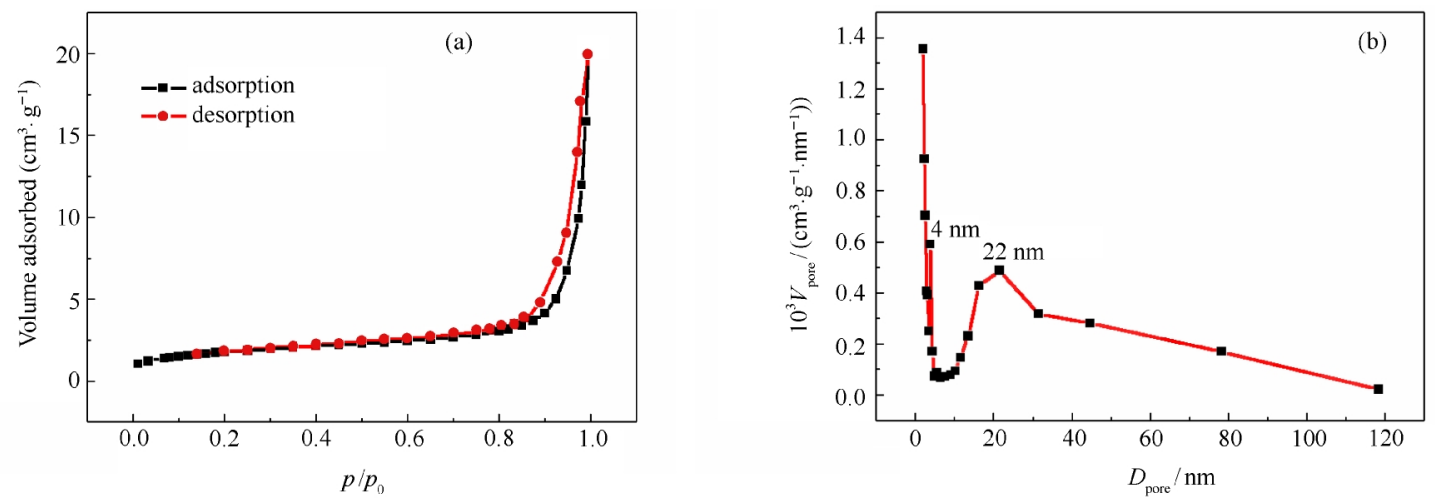

图 $6 \mathrm{WC}$ 样品的氮气吸附-脱附曲线 $(a)$ 和孔径分布 $(b)$

Fig.6 $\mathrm{N}_{2}$ adsorption-desorption curves (a) and pore diameter distribution (b) of WC sample

分布较宽. 通过计算分析得到该样品的比表面积 $S_{\mathrm{BET}}=6.44 \mathrm{~m}^{2} \cdot \mathrm{g}^{-1}$, 平均孔径 $D_{\mathrm{BJH}}=22.18 \mathrm{~nm}$.

\section{3 样品的电催化性能}

催化剂颗粒富含介孔或微孔是催化剂拥有较高 的比表面积的主要原因, 但由于孔径单一或者较小, 有可能导致大分子反应物无法进人颗粒内部, 因此 导致面积利用率较低 ${ }^{[17-18]}$. 对于大分子反应, 多孔径 分布的催化剂对降低传质阻力具有较好的前景. 另 一方面, 由于孔隙率增加, 组成催化剂颗粒的微观结 构单元尺寸降低到纳米级别, 不仅增大了催化剂的 反应表面积, 而且能使催化剂产生纳米尺寸效应, 从 而增加催化剂反应活性. 为研究本文制备的 WC 双 孔催化剂在大分子电催化反应的性能, 研究了质子 惰性介质中硝基苯在 $\mathrm{WC}$ 电极上的电化学行为. 作 为对比, 使用了未填充 WC 粉末的内嵌铂微盘电极 (Pt-MDE) 作为对比电极.

图 7 曲线 $\mathrm{a}$ 是 WC-PME 为工作电极, 在无 NB 的 TBAP/DMF 体系中的循环伏安曲线. 曲线 $b 、 c$ 分 别是 Pt-MDE 和 $\mathrm{WC}-\mathrm{PME}$ 在含 $0.03 \mathrm{~mol} \cdot \mathrm{L}^{-1}$ 硝基 苯的 TBAP/DMF 体系中的循环伏安曲线. 相比曲线 $a$, 曲线 $b$ 和 $c$ 都表现出了一对还原氧化峰. 这说明 铂和 $\mathrm{WC}$ 都对质子惰性体系下的 NB 有较好的电催 化还原作用. 进一步从图中可看出, 曲线 $\mathrm{c}$ 在 $-0.6-$ $-1.8 \mathrm{~V}$ 扫描电位区间出现了一对还原氧化峰, 还原 峰和氧化峰形状基本对称, 峰电流比 $I_{\mathrm{pd}} / I_{\mathrm{pa}}$ 约为 1 , 即说明该还原氧化反应为准可逆电荷跃迁过程, 电 化学步骤处于准平衡状态. 曲线 $\mathrm{c}$ 相比曲线 $\mathrm{b}$ 来讲 有更大的还原峰电流和较正的起峰电位和还原峰电 位, 可以说, 相比铂微盘电极的起峰电位, WC-PME 有着明显的优势, 且其峰电位比铂微盘电极正移了
$30 \mathrm{mV}$. 因此 WC-PME 表现出了更好的催化性能.

以上的现象表明, 除了 WC 在电催化硝基苯还 原反应中的良好性能外, 其结构因素也是提升性能 的一种方法. 因为在反应过程中, 溶质在催化剂表面 和内部的传质阻力的大小影响着整个反应的进行. 芳香族类硝基化合物的分子直径基本处于 0.5-0.8 $\mathrm{nm}$ 之间, 还原反应包括了硝基苯分子在溶液中运 动过程中的吸附, 较大的孔径有助于减小硝基苯分 子传质阻力, 从而增加其扩散速度和提升催化剂的 活性面利用率, 所以双孔催化剂有助于催化剂性能 的提升 ${ }^{[19]}$. 这也有可能是此双孔催化剂表现较高性 能的主要原因.

图8(a)为NB在DMF有机溶液中不同扫速下的循 环伏安曲线. 随着扫描速度的加快, 峰电流增大, 还

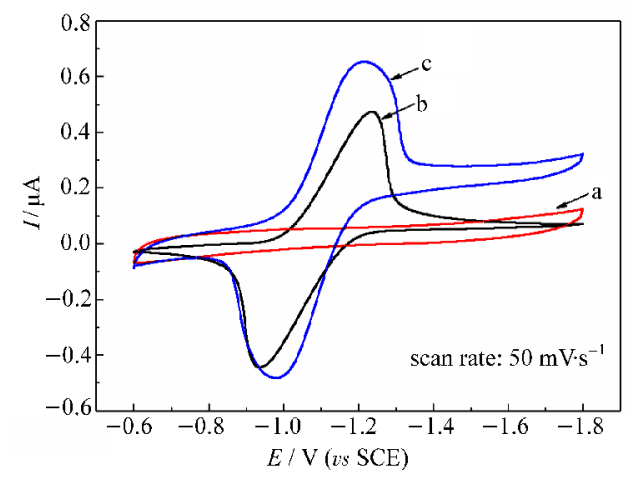

图 7 WC-PME 和 Pt-MDE 在不同溶液中的 循环伏安曲线

Fig.7 CVs of WC-PME and Pt-MDE in different solutions

(a) WC-PME in $0.1 \mathrm{~mol} \cdot \mathrm{L}^{-1} \mathrm{TBAP}$ in DMF solution; (b) Pt-MDE in $0.03 \mathrm{~mol} \cdot \mathrm{L}^{-1} \mathrm{NB}+0.1 \mathrm{~mol} \cdot \mathrm{L}^{-1} \mathrm{TBAP}$ in DMF solution; (c) WC-PME in $0.03 \mathrm{~mol} \cdot \mathrm{L}^{-1} \mathrm{NB}+0.1 \mathrm{~mol} \cdot \mathrm{L}^{-1} \mathrm{TBAP}$ in DMF solution; TBAP: $\mathrm{C}_{10} \mathrm{H}_{36} \mathrm{NClO}_{4} ; \mathrm{NB}: \mathrm{C}_{6} \mathrm{H}_{5} \mathrm{NO}_{2} ;$ MDE: micro disc electrode; DMF: $N, N$-dimethylformamide 

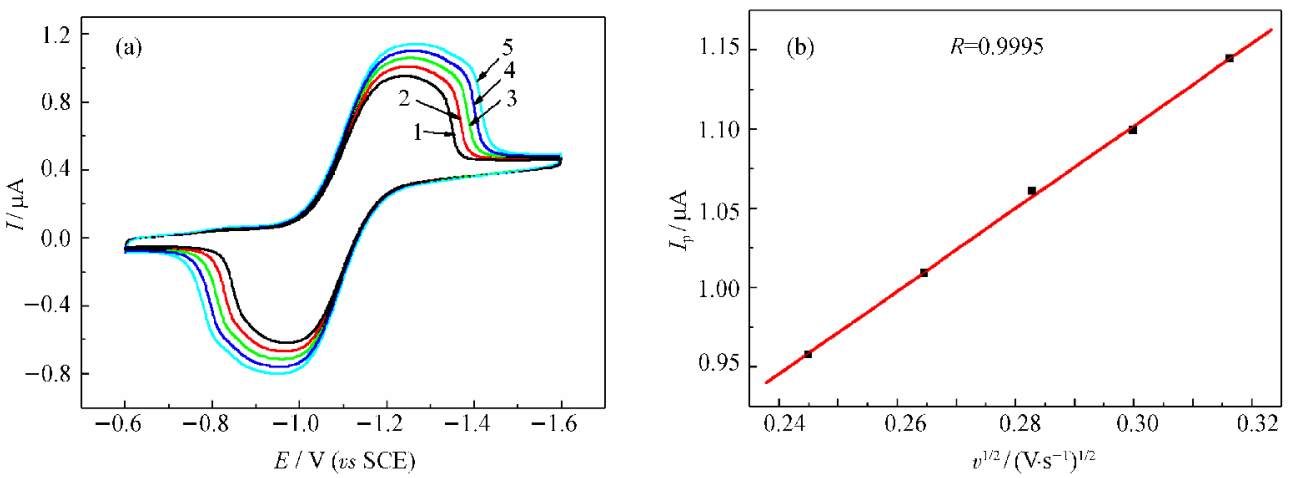

图 8 WC-PME 在硝基苯溶液中不同扫速下的循环伏安曲线(a)以及 $I_{\mathrm{p}}-v^{1 / 2}$ 的关系(b)

Fig.8 CVs of WC-PME in NB solution with different scanning rates (a) and the relation of $I_{\mathrm{p}}-v^{1 / 2}(\mathrm{~b})$ scan rate $\left(\mathrm{mV} \cdot \mathrm{s}^{-1}\right):(1) 60,(2) 70,(3) 80,(4) 90,(5) 100$

原峰电位负移, 氧化峰电位正移, 但氧化峰和还原峰 依然保持较好的可逆性, 图 8(b)为 $I_{\mathrm{p}}-v^{1 / 2}$ 关系拟合 直线, $R=0.9995$. NB 还原峰电流 $\left(I_{\mathrm{p}}\right)$ (以还原峰为例) 与扫描速率 $(v)$ 的平方根 $\left(v^{1 / 2}\right)$ 成正比, 说明 $\mathrm{NB}$ 在电 极界面的传质为线性扩散, 电极过程是由 NB 向电 极/溶液界面的扩散所控制.

\section{4 结 论}

以偏铇酸铵 (AMT) 为原料, 采用超声空化辅助 的方法对前驱体进行造粒和再分散, 并采用气-固反 应法制备了双孔隙介孔结构碳化铇(WC). WC 颗粒 呈介孔空心球状结构, 经二次超声分散后球状结构 破裂, 得到分布均匀的由纳米 WC 组成的 WC 微粒. 纳米 WC 颗粒均匀, 孔隙发达, 前后贯通. 空气中样 品在 $410{ }^{\circ} \mathrm{C}$ 以下保持稳定, 之后发生由双孔引起的 两次氧化反应. 从硝基苯的还原反应中可以看出, 相 比铂微盘电极, 双孔 WC 催化剂表现出了更好的电 催化活性, 其电极反应受扩散控制. 说明 WC 材料 在具有双孔纳米结构后表现出了更好的催化性能. 具体的传质过程和反应机理将在进一步的后续工作 中进行展开.

\section{References}

1 Levy, R. B.; Boudart, M. Science, 1973, 181: 547

2 Böhm, H. Nature, 1970, 227: 484

3 Ma, C. A.; Brandon, N.; Li, G. H. J. Phys. Chem. C, 2007, 111: 9504
$4 \quad$ Ma, C. A.; Huang, Y.; Tong, S. P.; Zhang, W. M. Acta Phys. Chim. Sin., 2005, 21: 721 [马淳安, 黄 烨, 童少平, 张维民. 物理化学学报, 2005, 21: 721]

5 Kojima, I.; Miyazaki, E.; Inoue, Y.; Yasumori, I. J. Catal., 1979, 59: 472

6 Horányi, G.; Rizmayer, E. M. React. Kinet. Catol. Lett., 1980, 12 21

7 Hara, Y.; Minami, N.; Matsumoto, H.; Itagaki, H. Appl. Catal. AGen., 2007, 332: 289

8 Vidick, B.; Lemaiter, J.; Leclercq, L. J. Catal., 1986, 99: 439

9 York, A. P. E.; Claridge, J. B.; Williams, V. C.; Brungs, A. J.; Sloan, J.; Hanif, A.; Al-Megren, H.; Green, M. L. H. Stud. Surf. Sci. Catal. B, 2000, 103: 989

10 Oyama, S. T.; Delporte, P.; Ham-Huu, C. P.; Ledoux, M. J. Chem. Lett., 1997: 949

11 Choi, S.; Thompson, L. T. Mater. Res. Soc. Symp. Proc., 1997, 454: 41

12 Moreno-Castilla, C.; Alvarez-Merino, M. A.; Carrasxo-Martin, F.; Fierro, J. L. G. Langmuir, 2001, 17: 1752

13 Ribeiro, F. H.; Boucart, M.; Dalla, B.; Ralph, A.; Iglesia, E. J. Catal., 1991, 130: 498

14 Yu, F. W.; Liu, H. Z.; Ji, J. B. Chem. Engin. Times, 2003, 17: 45 [于风文, 刘化章, 计建炳. 化工时刊, 2003, 17: 45]

15 Yao, Y. C.; Dai, Y. N.; Ren, H. L. Battery Bimonthly, 2004, 34: 250 [姚耀春, 戴永年, 任海伦. 电池, 2004, 34: 250]

16 Cachet-Vicier, C.; Vicier, V.; Cha, C. S. Electrochim. Acta, 2001, 47: 181

17 Sato, S.; Takahashi, R.; Sodesawa, T.; Koubata, M. Appl. Catal. AGen., 2005, 284: 247

18 Takahashi, R.; Sato, S.; Sodesawa, T.; Ikeda, T. Phys. Chem. Chem. Phys., 2003, 5: 2476

19 Caruso, R. A.; Antonietti, M. Adv. Funct. Mater., 2002, 12: 307 